\title{
Assessment of antimicrobial activity and cytotoxic effect of green mediated silver nanoparticles and its coating onto mini-implants
}

\author{
Swapna Sreenivasagan , Aravind Kumar Subramanian* and S. Rajeshkumar** \\ Department of Orthodontics, Saveetha Dental College, No. 162, Saveetha Institute of Medical and Technical Sciences, Saveetha University, \\ Chennai-600077, T.N., India \\ *Department of Orthodontics, Saveetha Dental College, No. 162, Saveetha Institute of Medical and Technical Sciences, Saveetha University, \\ Chennai-600077, T.N., India \\ **Department of Pharmacology, Saveetha Dental College, No. 162, Saveetha Institute of Medical and Technical Sciences, Saveetha University, \\ Chennai-600077, T.N., India
}

\section{Article Info}

Article history

Received 11 April 2020

Revised 25 May 2020

Accepted 27 May 2020

Published online 30 June 2020

Keywords

Silver nanoparticles

Chitosan

Mini-implant

Orthodontics

Antimicrobial

Cytotoxicity

\begin{abstract}
Mini-implants have become a major device in orthodontic treatment in this era, and practitioners intend to use for different clinical situations. Silver nanoparticle coating onto metal is known to reduce the colonization of bacteria, thus coating mini-implants will reduce the risk of peri-implantitis and implant failure. As there are new horizons being explored with nanotechnology, assessing its toxicity is very essential for its use in medicine. Chitosan based silver impregnated nanoparticles were synthesised using green tea extract and chitosan particles using thermal treatment. Formation of silver nanoparticles were assessed using double beam UV spectrophotometer. Mini-implants coated with silver nanoparticles were tested for antimicrobial property against Streptococcus mutans, Staphylococcus aureus, Lactobacillus and Candida albicans. Characterisation of the surface coating was done by using TEM. To assess the cytotoxic potential of silver nanoparticles, shrimp was cultured and were tested by adding various concentration of silver nanoparticle to it. UV-vis spectrophotometer was used to examine the formation of silver nanoparticles at 440 a.u peak. Transmission electron microscope (TEM) was used to characterize the nanoparticles that showed particles spherical in shape. A considerable zone of inhibition was also formed after $24 \mathrm{~h}$ of incubation for bacteria and $48 \mathrm{~h}$ of incubation of silver nanoparticles in Candida albicans. Cytotoxicity was assessed by testing on shrimp culture. Titanium mini-implants when coated with silver nanoparticles has excellent antimicrobial properties and, hence can be used a biomaterial in orthodontics but further tests are needed to evaluate the coating during and after placement.
\end{abstract}

\section{Introduction}

The use of mini-implants as an adjuvant in anchorage in orthodontic treatment of malocclusion has now been widely accepted in the present era. For anchorage control, various methods are used in practice that includes the bracket placement, anchorage from extraoral devices like headgear. The use of these type of anchorage requires maximum patient compliance and care (Tseng et al., 2006). Micro-screw mini-implants has been widely used because of its anchorage potential, easy placement, removal and low cost. Many factors influence the success of mini-implant like its placement, type of bone, placement procedure, angulation and site. To ensure success, it is important to prevent inflammation around the screw implants (Park et al., 2006). In orthodontic patient, there is a documented increase in Streptococcus and Lactobacillus (Pellegrini et al., 2009). Inflammation is caused as this is a site of injury, patient during initial days have poor maintenance of hygiene due to pain in the site, this acts as a nidus for the growth of micro-organisms.

Corresponding author: Dr. Swapna Sreenivasagan

Department of Orthodontics, Saveetha Dental College, No 162, Ponamallee High Road, Chennai-600077, T.N., India

E-mail: swapnasreenivasagan@gmail.com

Tel.: +91-9444406704

Copyright (C) 2020 Ukaaz Publications. All rights reserved.

Email: ukaaz@yahoo.com; Website: www.ukaazpublications.com
The use of nanoparticle coated material is now being accepted in dentistry as various benefits have been demonstrated by certain materials. In dentistry in general, silver nanoparticles are attempted to be incorporated into a wide range of materials because of its antimicrobial mechanism. Composites, adhesives, acrylic resin and even intra-radicular medicaments in endodontics are all the various materials that have previously been tried along with silver nanoparticles (Borzabadi-Farahani et al., 2014). Most of the studies are being conducted in an experiment to enhance the antimicrobial and the anti-cariogenic potential of the various components of the orthodontic materials (Borzabadi-Farahani et al., 2014). In orthodontics there has been previous studies incorporating silver nanoparticles in various materials like orthodontic, wires, adhesives, composite, brackets, bands, module, mini-implants in order to evaluate various properties like antibacterial effect, antiadherence, reduce friction and so on. Silver nanoparticle has shown bactericidal effect against gram negative micro-organisms. Silver is an unreactive component and has low ionization potential and is, thus also stable in aqueous solution, physiological fluids and solids. Silver nanoparticles have previously been coated onto hexagon dental implants and has shown positive antimicrobial activity against $S$. aureus and Candida (Rajeshkumar and Bharath, 2017). 
Chitosan poly (D-glucosamine) has been widely used for coagulation of metal ion removal as chitosan easily forms chelate with cation (Rajeshkumar and Bharath, 2017). Chitosan, a polysaccharide biopolymer derived from naturally occurring chitin, displays unique polycationic, chelating, and film-forming properties due to the presence of active amino and hydroxyl functional groups (Agarwal et al., 2017). A chitosan nanofiber scaffold can diminish infection in in vivo implantation due to its antibacterial properties (Kohsari et al., 2016).

There are various methods for coating and deposition on the miniimplants that have been used, the method ion include beam sputter deposition the substrate is bombarded by energetic ions beam becoming from inside an ion source; plasma ion etching method (Zegan et al., 2017). There is also coating done with high pressure $\mathrm{Hg}$ lamp in order for coating with various required concentrations (Zhao et al., 2009). Various methods are available for characterization of the nanoparticle and can be done using scanning electron microscope (SEM), transmission electron microscope (TEM) and atomic force microscope (AFM).

Studies have shown that from exposure to the oral cavity to both ionic and nanoparticle silver, there has been deposition in the oral epidermis, the glomeruli and in the intestines (Chang et al., 2006). There is also evidence in literature that there is a continuous release of silver ions from both silver nanoparticles and metallic silver (Kittler et al., 2010). The lower ionic strength medium of silver nanoparticles results in greater toxicity (Rajeshkumar and Malarkodi, 2014). Silver when administered through oral route is known absorbed in a range of $0.4-18 \%$ in mammalians and in man, a value of $18 \%$ absorption. Silver is distributed to all of the organs and highest levels are observed in the intestine and stomach. Silver toxicity in skin is expressed as argyria. Excretion of silver is via secretions of the bile and urine.

In the present age of technology there is wide range of studies that are undertaken to study the antimicrobial property of nanoparticles that are coated onto various materials that are used in the oral environment. It is evident that there is some antibacterial property in comparison to uncoated materials. The main objective of the study is to evaluate the peak of formation of silver nanoparticles under UV-vis spectrophotometer, to study the antibacterial activity against Streptococcus mutans, Lactobacillus and Staphylococcus aureus as these are the main bacteria found in increased levels in orthodontic patients and antifungal activity against Candida albicans. In this study we aim at synthesizing green mediated silver nanoparticles, coating it onto mini-implants, test for antimicrobial activity and to find the toxic potential of silver nanoparticles by placing them in shrimp culture at their naupulii stage.

\section{Materials and Methods}

\subsection{Synthesis of chitosan silver nanoparticles}

$0.5 \mathrm{~g}$ chitosan and $0.5 \mathrm{ml}$ of acetic acid was measured and added to $49.5 \mathrm{ml}$ of distilled water and allowed to mix using magnetic stirrer. $10 \mathrm{mM}$ of silver nitrate in $10 \mathrm{ml}$ of distilled water and these solutions were mixed by continuous stirring to promote dissolution. Green tea extract was prepared using boiling water. The extract solution of the plant solution was added to the aqueous silver nitrate solution and covered with aluminium foil and was subjected to magnetic stirring for the next $8 \mathrm{~h}$. All the containers were washed and rinsed with deionized water prior to use. The mixture was separated into test tubes and centrifuged at $8000 \mathrm{rpm}$ for $15 \mathrm{~min}$.

\subsection{Coating of titanium mini-implants}

Titanium mini-implants along with the solution containing silver nanoparticles was placed in a magnetic stirrer followed by heating and again stirring then, allow for the deposition of the particles on the mini-implants.

\subsection{UV vis spectroscopy and TEM analysis}

Formation of silver nanoparticles by reduction of $\mathrm{AgNO}_{3}$ in the chitosan solution was scanned between 250-700 a.u for every $1 \mathrm{~h}$ for the next $6 \mathrm{~h}$. The results were consistent with the typical spectra for this solution. The absorption peaks that are formed between 390 and 470 a.u are of those related to different clusters of silver ions (dos Santos et al., 2004). The surface morphology was assessed using TEM.

\subsection{Antimicrobial activity}

Antimicrobial activity of silver nanoparticles was performed by using agar well diffusion method. The muller Hinton agar for antibacterial and rose Bengal agar for antifungal (Candida albicans) was used. In order for the assessment of antimicrobial property was assessed after the silver nanoparticles were formed by placing 50, 100 and $150 \mu \mathrm{l}$ onto well cut in 4 petridishes and to test antimicrobial property against Streptococcous mutans, Staphylococcus aureus, Lactobacillus and Candida albicans. The result of antibacterial efficacy was examined after $24 \mathrm{~h}$ and antifungal results after $48 \mathrm{~h}$. After coating was completed, the coated mini-implants were examined comparing with uncoated implants to evaluate the antimicrobial efficacy against the same organism.

\subsection{Toxic testing}

Shrimp eggs were cultured using a culture containing iodine free salt in water and sodium bicarbonate added to this as a source of nutrition. Nauplii of the shrimp that were formed in $24 \mathrm{~h}$ were collected and added 10 in one well to a 6 well ELISA plate. Silver nanoparticles in varying concentrations of 5,10,15,20 and $25 \mu 1$ were added to each well and one well was used as a control. The survival of the nauplii was assessed after $24 \mathrm{~h}$.

\section{Results}

\subsection{Antimicrobial activity results}

The antibacterial activity of the synthesized silver nanoparticles was confirmed by the zone of inhibition formed against Staphylococcus aureus, Lactobacillus sp., Streptococcus mutans as shown Table 1, Figure 1 and Figure 7. Strong antibacterial activity was observed against Staphylococcus aureus, Lactobacillus, antibacterial activity was observed against Streptococcus mutans but the zone of inhibition was lesser than that observed in the other bacteria. The synthesized nanoparticles did not have a very strong antifungal activity. 
Table 2, Figure 2 and Figure 8 showed the zone of inhibition around the same micro-organisms in coated and uncoated mini-implants. The zone of inhibition was measured as length by breadth determined by the dimension of the mini-implants. There was no bacterial growth around the coated mini-implants, there was no inhibition of bacterial growth seen in the uncoated mini-implants. Maximum zone of inhibition was observed for Staphylococcus aureus and the least for Candida albicans.

Table 3 and Figure 3 showed the results of cytotoxicity tested and the death and inhibition of growth of the organisms was observed only in higher concentrations of the 20 and $25 \mu$ l. There was no cytotoxic potential observed in lower concentrations.

\subsection{Properties of silver nanoparticles}

Figure 4 depicts the visual observation of the addition of green tea extract, chitosan and silver nitrate, the solution initially was formed in light brown colour and later on continued magnetic stirring changed to dark brown colour. The results of UV vis spectroscopy is depicted in the graph in Figure 5 where the peak for silver nanoparticle formation is observed at a range of $250-700 \mathrm{~nm}$. The silver nanoparticles and silver nanoparticles impregnated chitosan showed peak between $420-460 \mathrm{~nm}$, confirms the silver nanoparticles synthesis. The characterisation of the surface morphology of the silver nanoparticles formed was studied under Transmission electron microscope as observed in Figure 6 which revealed that the particle size was formed of 30-35 nm and the particles were uniformly distributed and spherical, triangular and hexagonal in shape.

Table 1: Zone of inhibition by silver nanoparticles

\begin{tabular}{|l|l|l|l|}
\hline \multirow{2}{*}{ Bacteria/Fungi } & \multicolumn{4}{|l|}{ Zone of Inhibition (In mm) } \\
\cline { 2 - 4 } & $\mathbf{5 0} \mu \mathrm{l}$ & $\mathbf{1 0 0} \mu \mathrm{l}$ & $\mathbf{1 5 0} \mu \mathrm{l}$ \\
\hline Staphylococcus aureus & 9 & 18 & 20 \\
Lactobacillus & 30 & 32 & 40 \\
Streptococcus mutans & 14 & 15 & 20 \\
Candida albicans & 9 & 10 & 12 \\
\hline
\end{tabular}

Table 2: Length and breadth of zone of inhibition comparing silver nanoparticle coated with uncoated mini-implant (Control)

\begin{tabular}{|l|l|l|l|l|}
\hline \multirow{2}{*}{ Micro-organis m } & \multicolumn{2}{|l|}{ Zone of inhibition formed (In mm) } \\
\cline { 2 - 5 } & \multicolumn{2}{|l|}{ Coated Mini-implant } & \multicolumn{3}{|c|}{ Control } \\
\cline { 2 - 5 } & Length & Breadth & Length & Breadth \\
\hline Staphylococcus aureus & 11 & 8 & Nil & Nil \\
Lactobacillus sp. & 15 & 11 & Nil & Nil \\
Streptococcus mutans & 15 & 10 & Nil & Nil \\
Candida albicans & 12 & 6 & Nil & Nil \\
\hline
\end{tabular}

Table 3: Cytotoxicity: Assessing the number of live napulii after $24 \mathrm{~h}$

\begin{tabular}{|l|l|}
\hline $\begin{array}{l}\text { Concentration of silver } \\
\text { nanoparticle (In microlitres) }\end{array}$ & No. of live organisms \\
\hline 5 & 10 \\
10 & 10 \\
15 & 10 \\
20 & 8 \\
25 & 5 \\
\hline
\end{tabular}

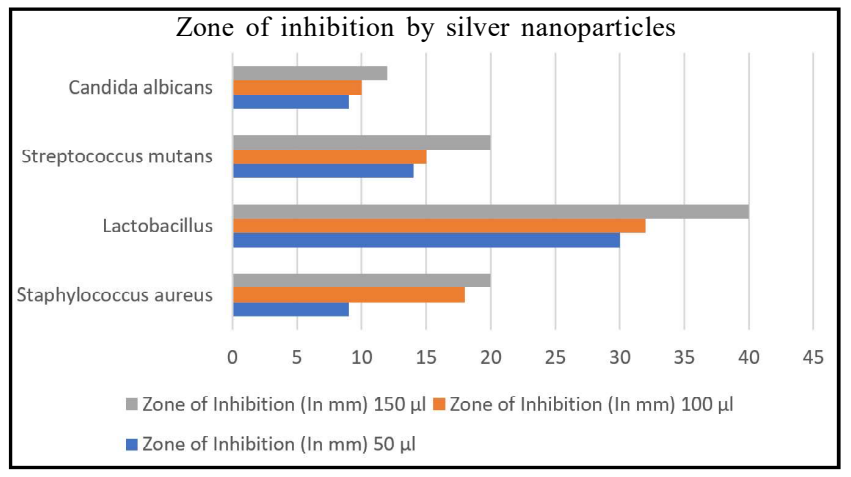

Figure 1: Zone of inhibition by silver nanoparticles.

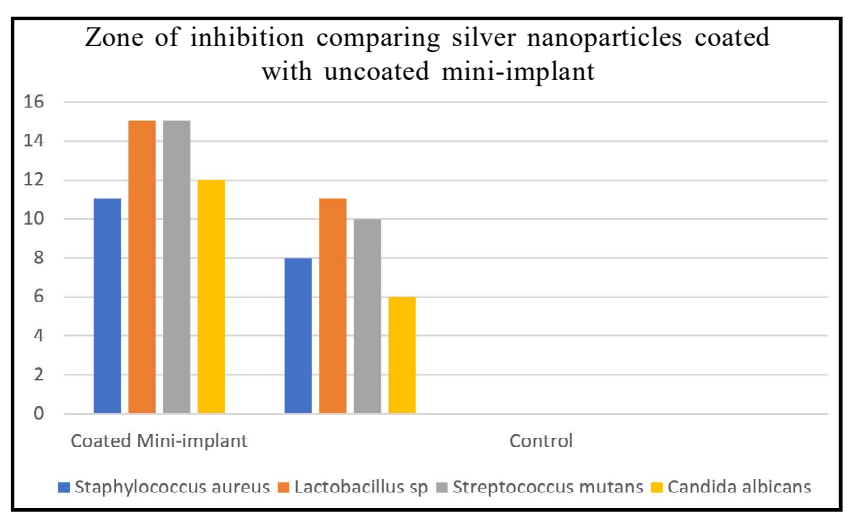

Figure 2: Length and breadth of zone of inhibition comparing silver nanoparticle coated with uncoated mini-implant (Control).

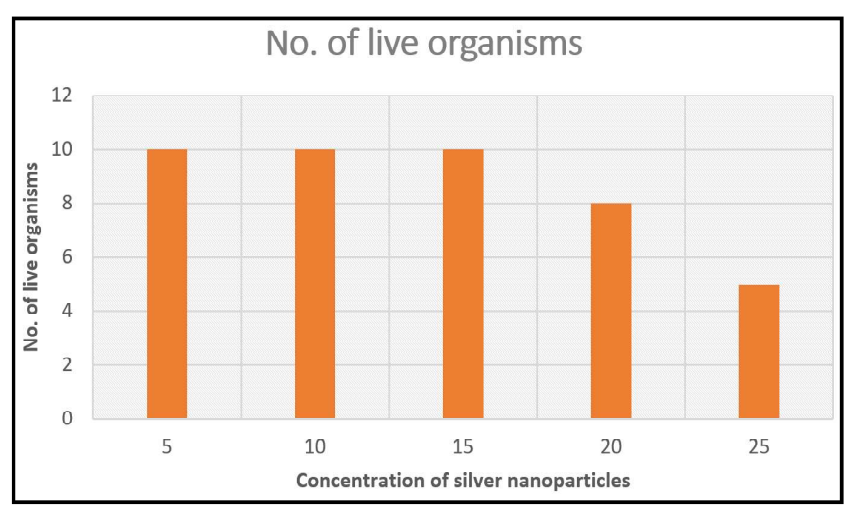

Figure 3: Assessing the number of live napulii after $24 \mathrm{~h}$.

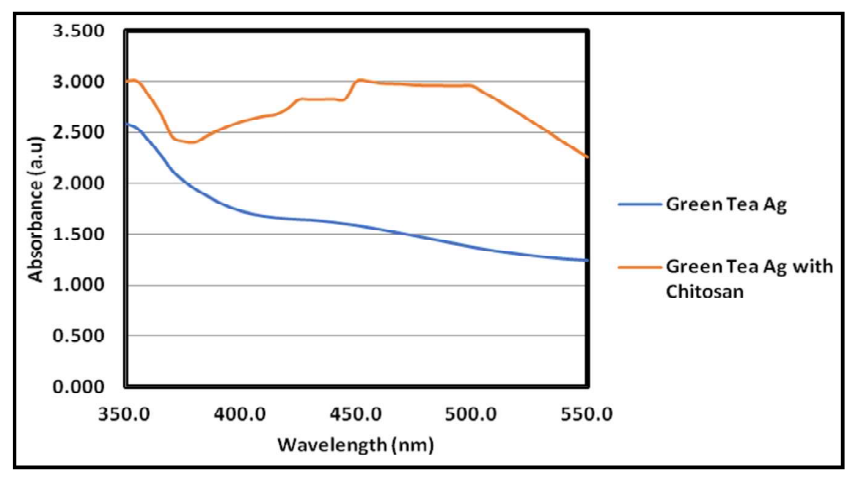

Figure 4: UV-vis spectroscopic analysis. 


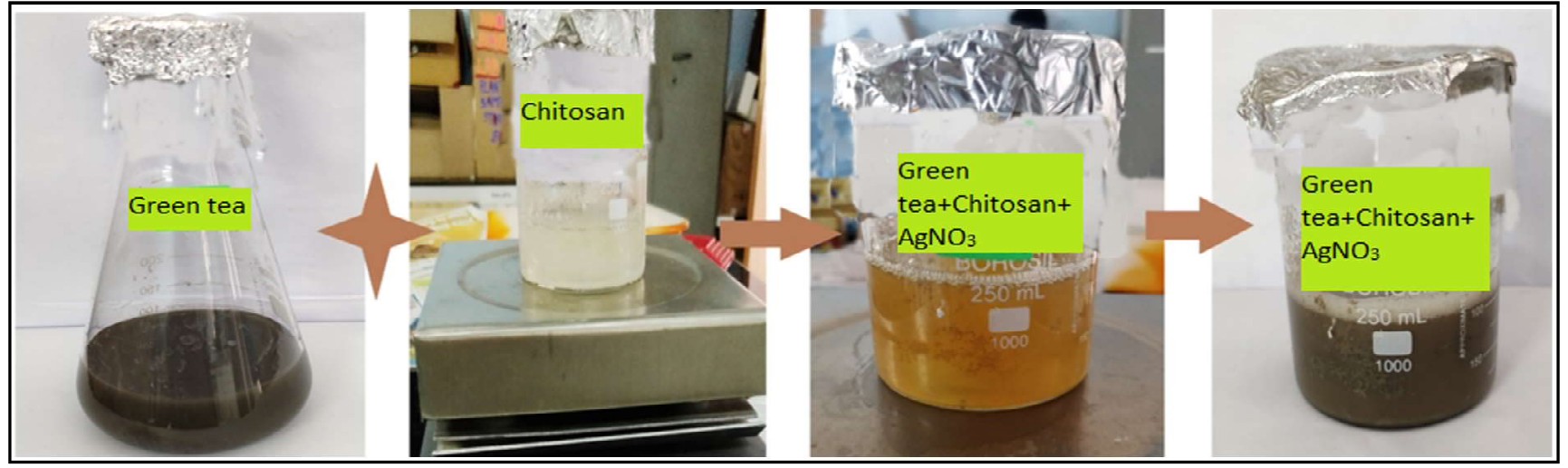

Figure 5: Visual observation.

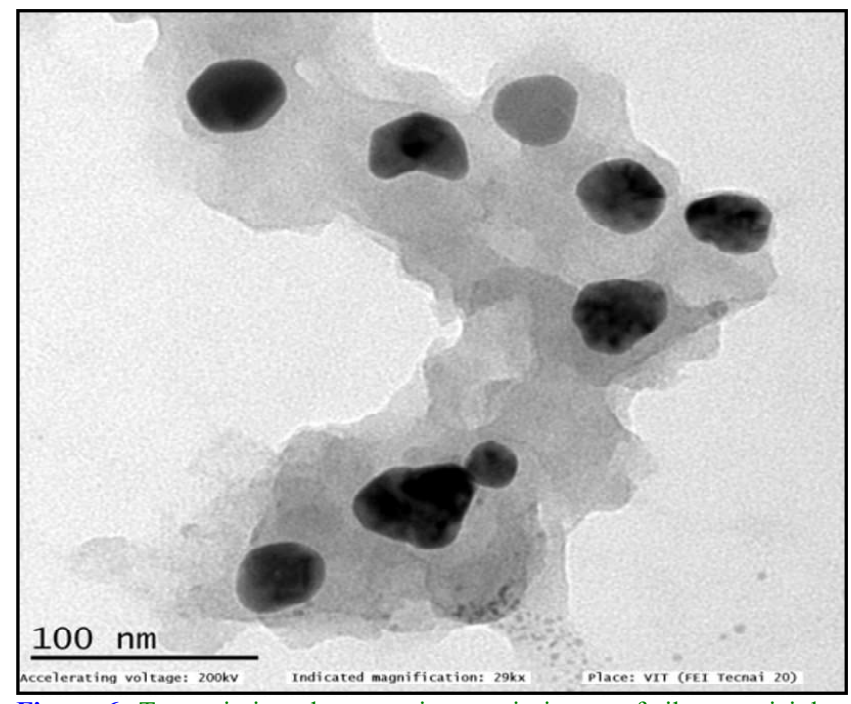

Figure 6: Transmission electron microscopic image of silver participles.

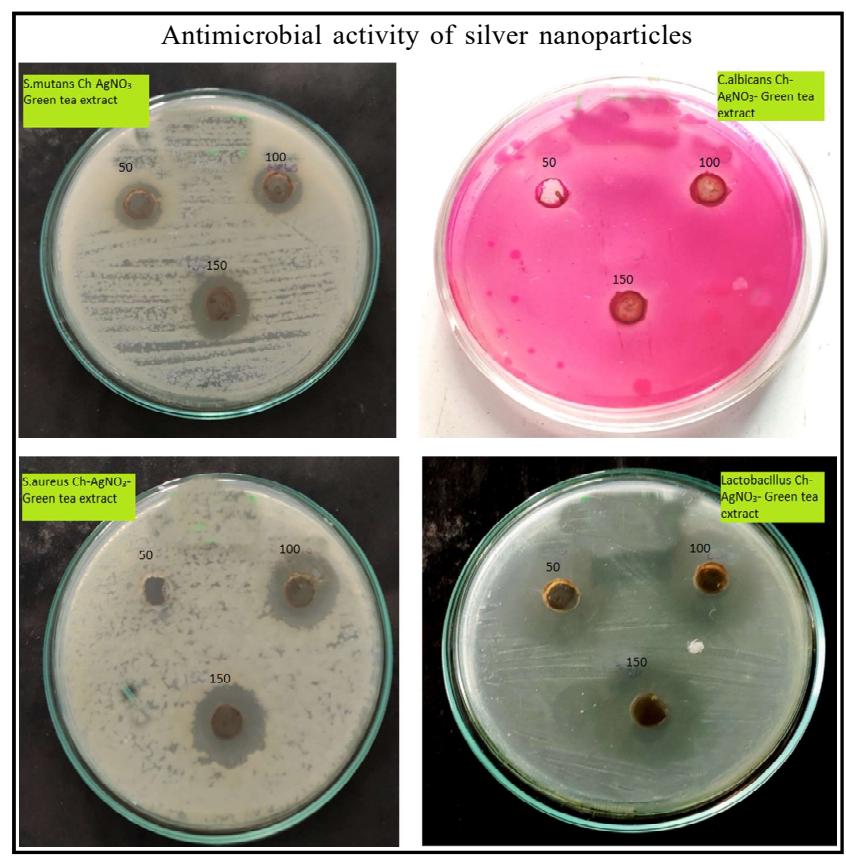

Figure 7: Antimicrobial activity of silver participles.
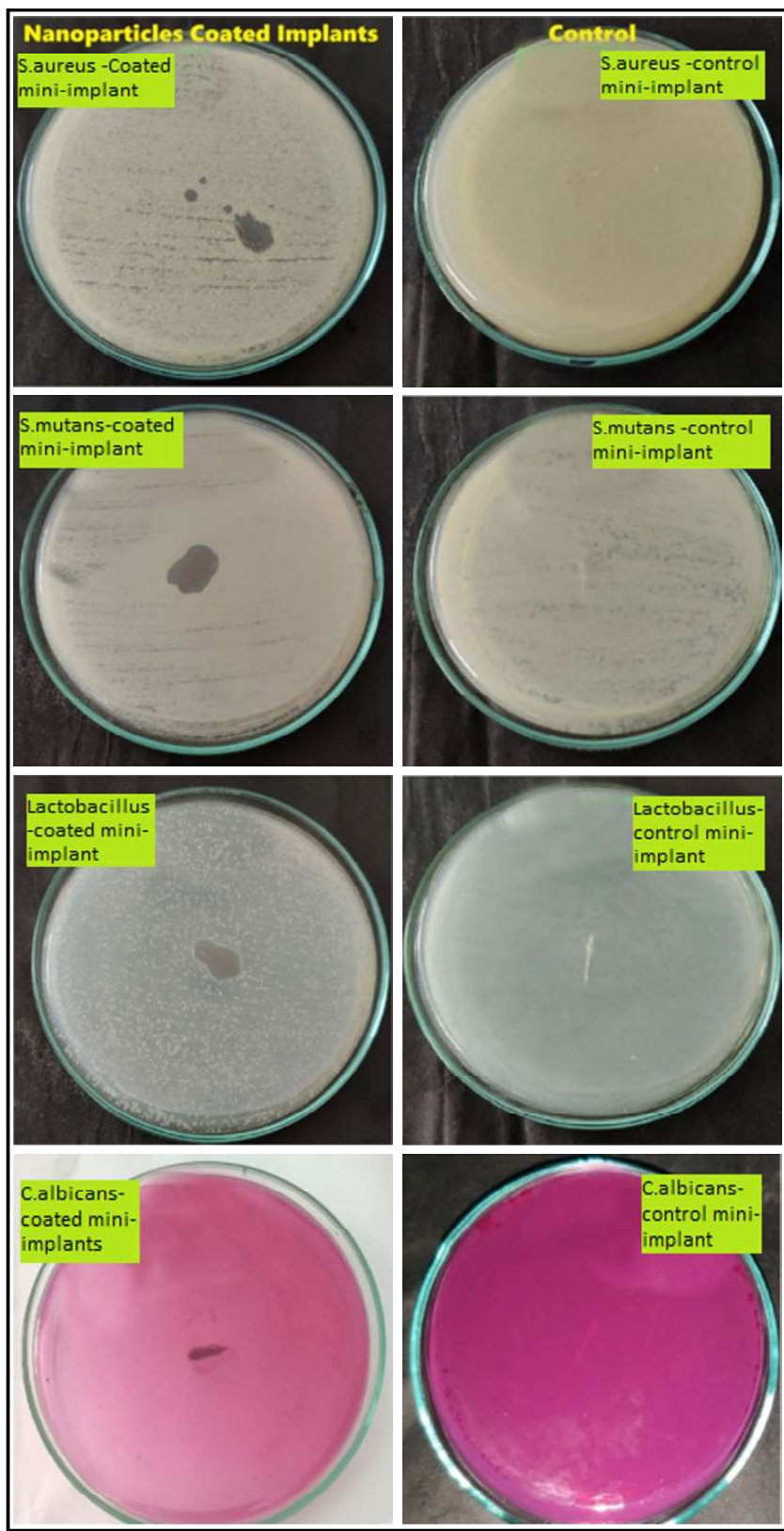

Figure 8: Zone of inhibition of mini-implants coated with nanoparticles. 


\section{Discussion}

Mini-implants are used for orthodontic anchorage and are usually loaded early to reduce treatment time and is removed after treatment. Mini-implants are placed near tooth roots (Park et al., 2006). The principle factor for stability is only by mechanical lock to the bone. Poor attention to oral hygiene lead to inflammation in the tissues around the mini-implants and hastened their loss (Tseng et al., 2006).

In this study, the silver nanoparticles that were formed, showed a peak of $450 \mathrm{~nm}$. The silver nanoparticles had good antibacterial effect even at low concentrations that increased as the concentration was increased. Antifungal activity as present that was better at higher concentration. TEM results showed that the silver nanoparticles formed were of spherical, triangular and hexagonal in shape and of the size of $30-35 \mathrm{~nm}$. The toxicity results showed that in lower concentrations all the nauplii survived, mild toxicity was seen in higher concentrations.

Silver nanoparticles has properties such as optical and catalytic properties, which depend on the size and shape of the produced nanoparticles (Khodashenas and Ghorbani, 2019). Silver nanoparticles have an antibacterial effect due to its activity of forming pits in the cell wall of the bacteria. There is an evident increase in the cell permeability that will result in cell death (Chaloupka et al., 2010). A chitosan nanofiber scaffold can diminish infection in in vivo implantation due to its anti-bacterial properties. Chitosan particles gives a positive charge to the nanoparticles, this will thus increase their binding to the negative charge of the bacterial cell wall (Hernández-Sierra et al., 2008). Chitosan films with silver nanoparticles antibacterial property against Escherisia coli for a longer time than pure chitosan films (Agarwal et al., 2017). The electrical and antibacterial properties of $\mathrm{CS} / \mathrm{AgNP}$ and $\mathrm{CS} / \mathrm{Ag}^{+}$ions bio nanocomposite depend on the concentration. Nanoparticles research has reached unique interest because of its properties from the bulk. Silver nanoparticles (Ag NPs) have gained substantial attention due to their potential applications in medical field especially in the production of biodegradable surgical sutures (Kohsari et al., 2016). Silver particles have the various reactions with a bacterial cell, that are interaction with cell wall causing lysis, preventing DNA replication and also disrupts bacterial protein synthesis (Chaloupka et al., 2010). Considering the liquid dilution method to find the minimum inhibition concentration of $25 \mathrm{~nm}$ AgNP is present against $S$. mutans and average of $4.8 \mu \mathrm{g} / \mathrm{mL}$ is also responsible for the excellent antimicrobial activity of silver nanoparticles (Hernández-Sierra et al., 2008).

Orthodontic wires coated with nickel-phosphorus film has shown to reduce the friction which is a major factor that restricts treatment (Redlich et al., 2008). Silver nanoparticle has been previously added to adhesive used for bracket placement and has demonstrated antimicrobial activity against S.mutans (Rajeshkumar and Bharath, 2017). In an in vitro study when silver nanoparticles was incorporated into the orthodontic band cement and antibacterial activity was present for 28 days (Moreira et al., 2014). Various nanoparticles have been coated onto orthodontic adhesive including silver, zinc oxide and titanium oxide. Silver nanoparticles were coated on modules and exhibited increased strength than conventional modules (Hernández-Gómora et al., 2017). Titanium nanoparticle incorporation in composite revealed lowering the colonisation of
S. mutans, S. sanguis and L. acidophilus and the shear bond strength of the composite was reduced by still under acceptable levels (Sodagar et al., 2016). In the field of implantology, nanoparticles are deposited onto titanium mini-implants and has shown to be advantageous for both cell proliferation and to prevent infection from micro-organisms. Thermal annealing method in air had been used to produce a thin film which was stable even in an aqueous environment (Zegan et al., 2017). Bacterial adhesion onto titanium implant is one of the major factor, hence surface modification by coating with metallic nanoparticles will cause a decrease in the colony forming units of bacteria and will be more beneficial for treatment (García Contreras et al., 2011). Silver nanoparticles coated onto orthodontic wires and brackets showed anti-adherence properties of strains of Streptococcus mutans even in the presence of orthodontic appliances (Espinosa-Cristóbal et al., 2018). As white spot lesions are a known side effect to orthodontic treatment, silver nanoparticles were also attempted to be used along with orthodontic composite. Transbond as significant antibacterial activity was demonstrated along against $S$. mutans (Sodagar et al., 2016). On coating titanium mini-implants with biopolymer silver nanoparticles, significant antimicrobial property has been demonstrated against $S$. mutans, S. sanguinis, and $A$. actinomycetemcometans. In situ release and good biocompatibility was achieved in a previous study when silver nanoparticles were incorporated into band cement that also had good antimicrobial activity (Moreira et al., 2014).

Silver nitrate when at concentrations of $24 \mathrm{mM}$ (corresponding to $308 \mathrm{mg}$ of silver/kg of body weight) when present in the drinking water can induce death over few days (Salhab et al., 1998). The effects of particulate silver are mediated via silver ions releasing from the particle surface (Hadrup and Lam et al., 2014). The potential sources of AgNPs includes leaching of intact particles from consumer products, disposal of waste from industrial processes, intentional release into contaminated waters, and the natural formation of AgNPs in surface and ground water (Sharma et al., 2019). The aggregation of nanoparticle have an effect on toxicity by reducing the dissolution rate and uptake by organisms and also the stability of these nanoparticles (Yang et al., 2012). The colloidal spherical silver nanoparticles and nano prisms are not genotoxic whereas silver nitrate is genotoxic even at $10 \mu \mathrm{g} / \mathrm{ml}$. Oxidative dissolution in experimental conditions (maximally $15 \%$ in $24 \mathrm{~h}$ ) is the key to the toxicity of most Ag NPs, highlights a critical role for dissolved silver complexed with thiols in the toxicity of all tested Ag NPs (Yang et al., 2012).

\section{Conclusion}

Chitosan-silver nanoparticles have very strong antibacterial activity against Staphylococcus aureus, Lactobacillus, antibacterial activity was observed against Streptococcus mutans was minimal and the synthesised nanoparticles did not have strong antifungal activity. The precipitate shows peak between $420-460 \mathrm{~nm}$, confirming the silver nanoparticles synthesis. Further, studies are needed to evaluate the long-term stability of the nanoparticle coating, the integrity of the mechanical property of the materials coated and the probable clinical use to reduce bacterial adherence and periimplantitis that are caused by oral micro-organisms. A limitation of this study is that its results are only in vitro and toxicity needs in to be checked in higher living organisms. 


\section{Acknowledgements}

We sincerely thank Indian Orthodontic Society Research Fund - Dr. C. Padmalatha Memorial Grant for Research (Student Members) for funding this study. We sincerely thank favanchor company for providing their mini-implants for purpose of this study. We also sincerely thank Ms M.Tarani for helping with the laboratory procedures.

\section{Conflict of interest}

The authors declare that there are no conflicts of interest in the course of conducting the research. All the authors had final decision regarding the manuscript and decision to submit the findings for publication.

\section{References}

Agarwal. H, Kumar, S.V. and Rajeshkumar, S. (2017). Areview on green synthesis of zinc oxide nanoparticles: An eco-friendly approach. ResourceEfficient Technologies, 3(4):406-413.

Borzabadi-Farahani, A.; Borzabadi, E. and Lynch, E. (2014). Nanoparticles in orthodontics, a review of antimicrobial and anti-caries applications. Acta. Odontol. Scand., 72:413-417.

Chaloupka, K.; Malam, Y. and Seifalian, A.M. (2010). Nanosilver as a new generation of nanoproduct in biomedical applications. Trends Biotechnol., 28:580-588.

Chang, A.L.S.; Khosravi, V. and Egbert, B. (2006). A case of argyria after colloidal silver ingestion. J. Cutan. Pathol., 33:809-811.

dos Santos, Goulet.; P.J.G., Pieczonka.; N.P.W., Oliveira. and Aroca, R.F (2004). Gold nanoparticle embedded, self-sustained chitosan films as substrates for surface-enhanced raman scattering. Langmuir, 20: 10273-10277.

Espinosa-Cristóbal, L.F.; López-Ruiz, N.; Cabada-Tarín, D.; Reyes-López, S.Y.; Zaragoza-Contreras, A.; Constandse-Cortéz, D.; Donohué-Cornejo, A.; Tovar-Carrillo, K.; Cuevas-González, J.C. and Kobayashi, T. (2018). Antiadherence and antimicrobial properties of silver nanoparticles against streptococcus mutans on brackets and wires used for orthodontic treatments [WWW Document]. J. Nanomater.

García Contreras, R.; Argueta Figueroa, L.; Mejía Rubalcava, C.; Jiménez Martínez, R.; Cuevas Guajardo, S.; Sánchez Reyna, P.A. and Mendieta Zeron, H. (2011). Perspectives for the use of silver nanoparticles in dental practice. Int. Dent. J., 61:297-301.

Hadrup, N. and Lam, H.R. (2014). Oral toxicity of silver ions, silver nanoparticles and colloidal silver: A review. Regul. Toxicol. Pharmacol., 68:1-7.

Hernández-Gómora. A.E.; Lara-Carrillo, E.; Robles-Navarro, J.B.; ScougallVilchis, R.J.; Hernández-López, S.; Medina-Solís, C.E. and Morales-Luckie, R.A. (2017). Biosynthesis of silver nanoparticles on orthodontic elastomeric modules: Evaluation of mechanical and antibacterial properties. Molecules, 22:1407.

Hernández-Sierra, J.F.; Ruiz, F.; Cruz Pena, D.C.; Martínez-Gutiérrez, F.; Martínez, A.E.; de Jesús Pozos Guillén, A.; Tapia-Pérez, H. and Martínez Castañón, G. (2008). The antimicrobial sensitivity of Streptococcus mutans to nanoparticles of silver, zinc oxide, and gold. Nanomedicine Nanotechnol. Biol. Med., 4:237-240.

Kittler, S.; Greulich, C.; Diendorf, J.; Köller, M. and Epple, M. (2010). Toxicity of silver nanoparticles increases during storage because of slow dissolution under release of silver ions. Chem. Mater., 22:4548-4554.
Khodashenas, B. and Ghorbani, H.R. (2019). Synthesis of silver nanoparticles with different shapes. Arabian Journal of Chemistry, 12(8):1823-1838.

Kohsari, I.; Shariatinia, Z. and Pourmortazavi, S.M. (2016). Antibacterial electrospun chitosan-polyethylene oxide nanocomposite mats containing bioactive silver nanoparticles. Carbohydr. Polym., 140: 287-298.

Moreira, D.M.; Oei, J.; Rawls, H.R.; Wagner, J.; Chu, L., Li, Y. Zhang, W. and Whang, K. (2014). A novel antimicrobial orthodontic band cement with in situ-generated silver nanoparticles. Angle Orthod., 85:175-183.

Park, H.S.; Jeong, S.H. and Kwon, O.W. (2006). Factors affecting the clinical success of screw implants used as orthodontic anchorage. Am. J. Orthod. Dentofacial Orthop., 130:18-25.

Pellegrini, P.; Sauerwein, R.; Finlayson, T.; McLeod, J., Covell, D.A.; Maier, T. and Machida, C.A. (2009). Plaque retention by self-ligating vs elastomeric orthodontic brackets: Quantitative comparison of oral bacteria and detection with adenosine triphosphate-driven bioluminescence. Am. J. Orthod. Dentofacial Orthop., 135:426.e1-426.e9.

Rajeshkumar, S. and Malarkodi, C. (2014). In vitro antibacterial activity and mechanism of silver nanoparticles against foodborne pathogens. Bioinorganic Chemistry and Applications.

Rajeshkumar, S. and Bharath, L.V. (2017). Mechanism of plant-mediated synthesis of silver nanoparticles: A review on biomolecules involved, characterisation and antibacterial activity. ChemicoBiological Interactions, 273:219-227.

Redlich, M.; Katz, A.; Rapoport, L.; Wagner, H.D.; Feldman, Y. and Tenne, R. (2008). Improved orthodontic stainless steel wires coated with inorganic fullerene-like nanoparticles of WS2 impregnated in electroless nickel-phosphorous film. Dent. Mater., 24:1640-1646.

Salhab, S.O.T.S.M.Z.M.N.G.M.S.S.A.S. (1998). Toxicity of a new antismoking mouthwash 881010 in rats and rabbits. J. Toxicol. Environ. Health A, 53:47-60.

Sharma, V.K.; Sayes, C.M.; Guo, B.; Pillai, S.; Parsons, J.G.; Wang, C.: Yan, B. and $M a, X$. (2019). Interactions between silver nanoparticles and other metal nanoparticles under environmentally relevant conditions: A review. Science of The Total Environment, 653:1042-1051.

Sodagar, A.; Akhavan, A.; Hashemi, E.; Arab, S.; Pourhajibagher, M.; Sodagar, K.; Kharrazifard, M.J. and Bahador, A. (2016). Evaluation of the antibacterial activity of a conventional orthodontic composite containing silver/hydroxyapatite nanoparticles. Prog. Orthod., 17:40.

Tseng, Y.C.; Hsieh, C.H.; Chen, C.H.; Shen, Y.S.; Huang, I.Y. and Chen, C.M. (2006). The application of mini-implants for orthodontic anchorage. Int. J. Oral Maxillofac. Surg., 35:704-707.

Yang, X.; Gondikas, A.P.; Marinakos, S.M.; Auffan, M.; Liu, J.; Hsu-Kim, H. and Meyer, J.N. (2012). Mechanism of silver nanoparticle toxicity is dependent on dissolved silver and surface coating in caenorhabditis elegans. Environ. Sci. Technol., 46:1119-1127.

Zegan, G.; Carausu, E.M.; Golovcencu, L.; Botezatu, A.S.; Cernei, E.R. and Anistoroaei, D. (2017). Nanoparticles deposition on mini-implants for osseo-integration and antibacterial properties improvement. Rev. Chim., 68:12.

Zhao, L.; Chu, P.K.; Zhang, Y. and Wu, Z. (2009). Antibacterial coatings on titanium implants. J. Biomed. Mater. Res. B Appl. Biomater., 91(B):470-480.

Citation: Swapna Sreenivasagan, Aravind Kumar Subramanian and S. Rajeshkumar (2020). Assessment of antimicrobial effects and cytotoxic effect of green mediated silver nanoparticles and its coating onto mini-implants. Ann. Phytomed., 9(1):207-212. http://dx.doi.org/10.21276/ap.2020.9.1.27 